\title{
BASE Y FUNDAMENTO EN EL PROCESO Y RESULTADO DE LA INVESTIGACIÓN EDUCACIONAL
}

Felipe Aguirre Chávez

\section{INTRODUCCIÓN}

En el desarrollo histórico social, las categorías base y fundamento conforman dos cuestiones diferentes, pero a su vez, intrínsecamente, conectadas. No hay objeto, campo o dimensión social fuera de su alcance. Tal vez, metafóricamente hablando, base y fundamento conforman el sello y la cara de una misma moneda.

Las variables, categorías, conceptos, definiciones, supuestos, juicios, razonamientos y argumentos están determinados por sus bases y fundamentos.

En el presente artículo pretendemos responder a las cuestiones ¿A qué llamamos base? y ¿A qué llamamos fundamento? Dos cuestiones esenciales importantes para la investigación científica educacional.

\section{Estado del problema}

En el magisterio nacional hay dos falencias recurrentes: el empirismo, y la dispersión teórica que, en gran parte, obstaculizan el proceso de producción de conocimientos científicos y, por ende, el diseño de alternativas viables de solución de problemas de la práctica.

Bueno es saber que el Ministerio de Educación, en los últimos años, ha puesto en marcha masivos planes y programas de formación en servicio: 


\section{Tabla 1}

Programas de capacitación docente

\begin{tabular}{|c|c|c|c|}
\hline $1995-2001$ & $2002-2006$ & $2007-2012$ & 2008-2012 \\
\hline $\begin{array}{l}\text { Plan Nacional de } \\
\text { Capacitación Docente } \\
\text { (PLANCAD) }\end{array}$ & $\begin{array}{l}\text { Programa Nacional } \\
\text { de Formación de } \\
\text { Docentes en Servicio } \\
\text { (PNFS) }\end{array}$ & $\begin{array}{l}\text { Programa de Formación y } \\
\text { Capacitación Permanente } \\
\text { (PRONAFCAP) }\end{array}$ & $\begin{array}{l}\text { Programa Estratégico } \\
\text { de Logros de } \\
\text { Aprendizaje. } \\
\text { (PELA) }\end{array}$ \\
\hline
\end{tabular}

Fuente: elaboración propia

Los planes y programas compartieron ciertas similitudes. Las particularidades más saltantes están referidas a los contenidos de las propuestas de capacitación centradas en aspectos metodológicos y de especialización. Así, de los cuatro, tres (PLANCAD, PNFS y PELA) estuvieron centrados en cuestiones metodológicas, (métodos, estrategias, técnicas, procedimientos) y PRONAFCAP aunque tenía como eje especializar al docente en el proceso, se disolvió y, como refiere Cuenca (2012:13): "tuvo como objetivo mejorar las capacidades, conocimientos, actitudes y valores para el desempeño docente", pero no fue sostenible ni compacto en sus planteamientos.

En todo caso, en materia de capacitación se han priorizado aspectos metodológicos descuidando aspectos teóricos en unidad de la práctica y concordando con Rivera (2010:6), al docente: ". . . No se le enseñan las bases científicas de la técnica, ni tampoco los fines u objetivos de las mismas" y como refiere, Leblond, (2010:6): "Los profesionales en la ciencia son cada vez más técnicos y menos intelectuales, y los momentos de creación y crítica no se dan tanto en la educación escolar como en la educación universitaria". Es decir, la situación por la que atraviesa la educación peruana, requiere, además, desarrollar en sus maestros habilidades metodológicas, habilidades y competencias académicas y teóricas que les posibiliten diseñar propuestas para construir nuevos conocimientos y contribuir en la solución de problemáticas y falencias del proceso educativo y del proceso de enseñanza y aprendizaje.

En esa perspectiva, en el año 2012 el Ministerio de Educación ha publicado el documento Marco del Buen Desempeño Docente (MBDD), en el que por primera vez en la historia de la educación peruana se pone énfasis en los enfoques, aspectos teóricos, metodológicos del diagnóstico y éticos de la profesión docente. Asimismo, presenta los criterios sobre lo que es una buena enseñanza desde sus dos pilares: base y fundamento. 


\section{2. ¿Qué entendemos por base?}

Como base se asumen las condiciones físicas y el contexto histórico-social en el que vive y se desarrolla el ser humano. Según Castro (2005, p. 491), "Es la forma históricamente condicionada mediante la cual la sociedad obtiene los bienes materiales y espirituales necesarios para su existencia". Sobre la base material se sostiene y garantiza la satisfacción de las necesidades primarias. Exceptuada de ella, es decir, de la base, la vida social sería inconcebible. Y si por alguna razón la actividad productiva cesara, entonces desaparecería la sociedad humana, se desintegrarían las instituciones y las organizaciones sociales, se extinguiría la cultura y se destruirán las formas habituales de existencia humana. Los hombres experimentarían un proceso regresivo hacia el estado primigenio de la civilización.

La base funge de plataforma material y, por ende, es la premisa de desarrollo social. Es el asiento sobre el que se configura el desarrollo humano en sus múltiples factores. A manera de ilustración, en Psicología es la estructura sobre la que se desarrollan las funciones psíquicas superiores; en Sociología constituye la plataforma económica que determina las relaciones sociales.

Howar Selsan (1990) indica que la base en términos más amplios es el mundo objetivo, un mundo que existe independientemente de la conciencia humana. Hemos nacido en ese mundo que no hemos creado y que permanecerá después de que nosotros desaparezcamos. Ese mundo objetivo con la totalidad de sus factores materiales y espirituales propios de un tiempo y contexto constituye la base.

\section{3. ¿Qué entendemos por fundamento?}

El presupuesto teórico que en el sentido más amplio abarca la concepción del mundo, la filosofía, los enfoques, las teorías que, eventualmente, orientan el punto de vista, el modo de ver y vivir la vida en la totalidad de sus factores de denomina fundamento. Es un aditamento ideológico que le confiere sentido a la existencia humana. Ayuda a explicar por qué determinados seres humanos son optimistas y otros pesimistas, produciéndose así lógicas diferencias de describir, explicar, comprender, dominar y transformar, incluso los mismos objetos y fenómenos de una misma realidad. Todo el aditamento conceptual con sus diferentes nominaciones que posee un sujeto, en su sentido más amplio se denomina, según la filosofía, concepción del mundo. Así, según Lefebvre (1968) "posee un sentido más amplio que la palabra filosofía" (p.7). En nuestro modesto modo de entender dos razones justifican su aseveración: en primer lugar, toda concepción del mundo implica una acción, es decir, algo más que una actitud filosófica; en segundo lugar, una concepción del mundo no es necesariamente la obra de tal o cual pensador, es más bien la obra y expresión de una época de la sociedad. 
En todo caso, desprovisto de base y fundamento, la vida social sería inconcebible. La base, en términos económicos, al ser las fuerzas productivas constituyen el aspecto físico de la realidad y las relaciones de producción conforman los aspectos cognoscitivos, ideológicos-culturales. Estos dos aspectos son la forma histórica condicionada mediante el cual la sociedad obtiene los bienes materiales y espirituales necesarios para su existencia y desarrollo. La interrelación interactiva de las fuerzas productivas y las relaciones de producción conforman la estructura, sobre la que se edifican las superestructuras, las instituciones, las concepciones políticas y jurídicas, la filosofía y la ciencia, el arte y la cultura; en una palabra: el fundamento.

\section{Relación intrínseca entre base y fundamento}

Hay relaciones intrínsecas, entre base y fundamento. Eventualmente las líneas delgadas de diferenciación podrían llevarnos a no distinguirlas.

Se trata de dos categorías unidas, aunque cualitativamente diferenciadas. En esencia conforman una dupla irrompible, recíprocos. Un ligero análisis nos puede llevar a no identificarlos: base y fundamento, aunque se encuentran unidos, son al mismo tiempo diferentes. En palabras de Castro (2005, p. 491): "Del modo de producción dependen las estructuras, las funciones y el desarrollo".

La psicología evolutiva tiene importantes aportaciones hacia la comprensión de las categorías de base y fundamento. En el desarrollo ontogénico, identifica la existencia de dos aspectos diferentes, pero a su vez intrínsecamente complementarias: lo biológico y lo social. Lo biológico es la parte física, la base material, la estructura necesaria e imprescindible, sin el cual el desarrollo de las funciones psíquicas superiores, los fundamentos no sería posible. Sobre la base de lo biológico y social se configura, gradualmente, la personalidad. Y, si el ser humano, eventualmente, es considerado el más grande y mejor ser evolucionado, se debe principalmente a su desarrollo cerebral. El cerebro humano tiene como función formidable el pensamiento, cuyo núcleo es el conocimiento. Merced a la interacción creadora de los factores biológicos y sociales en contextos históricamente definidos, desde un proceso intencional de abstracción de la realidad, el ser humano produce conocimiento sistematizado racionalmente como pensamiento para volver necesariamente a su origen, la práctica y desde ella a la teoría; y así sucesivamente, en movimiento cognoscitivo, en espiral, hacia el infinito.

En la misma línea de interrelación dialéctica de lo biológico y social destaca la interacción entre estructura y función. La estructura material celular con sus componentes anatómicosfisiológicos-químicos denominados aptitudes es definida por Castro, (2005, p.69) como "conjunto de condiciones psicofisiológicas individuales que constituyen las premisas 
naturales para la formación y desarrollo de las capacidades". Sobre la base de las aptitudes, por la interacción social, se desarrollan las funciones psíquicas superiores, (pensamiento, lenguaje y memoria), que reciben el nombre de capacidades. Lo valioso es saber que entre aptitud y capacidad existe recíproca interdependencia. Para un educador es muy importante saber esa interrelación, por cuanto la dotación genética y las funciones psíquicas superiores se interpenetran y determinan el desarrollo humano. Según la tesis histórica dialéctica materialista la interrelación entre aptitud y capacidad es de ida y vuelta. Las aptitudes determinan a las funciones y estas a su vez modifican, transforman a las aptitudes. Podemos entonces hablar de que "la función hace al órgano", pero las funciones se especializan sobre la calidad de las predisposiciones anatómicas y fisiológicas de los órganos. Y, a la inversa, los órganos que no se estimulan y desarrollan, los que no se ejercitan, mueren por desuso.

Para los educadores es importante saber que no existen puntos de vista educativos ni criterios pedagógicos y didácticos desprovistos de base y fundamento. La educación responde directa o indirectamente a intereses y necesidades de una sociedad en correspondencia al carácter de las relaciones de producción, su concepción e ideología prevalente. Educacionalmente hablando, hay dos formas cualitativamente diferenciadas de educar al ciudadano; florma más fácil y oficial es la de formar seres humanos para adecuarse y subordinarse al sistema oficial con su modo de producción y su ideología prevalente; la otra vía, metafóricamente hablando es: "nadar contra corriente", es formar personalidades con pensamientos y sentimientos de transformación, de cambio.

Las eventuales reformas educativas ocurridas en el Perú, por lo general, han terminado en desastre, por cuanto no partieron del conocimiento real de las bases económicas, sociales e históricas; y, al desconocimiento de las bases se correlaciona el desacierto de las propuestas y alternativas de eventuales intentos fallidos de cambio. Los dirigentes del sistema educativo peruano, al no partir de la realidad, por medio de diagnósticos científicos o al caer en el excesivo abuso de supuestos, juzgaron la destartalada educación peruana, no desde los hechos mismos sino desde puntos de vista de compilaciones sesgadas de datos estadísticos que al pueblo le cuesta millonarios gastos. Con datos muchas veces fraudulentos, se tejieron propuestas confinadas al fracaso. En otros casos, del erario se han gastado exorbitantes sumas de dinero en diagnósticos abordados con instrumentos con ínfima validez y confiabilidad, desprovistos de fundamento teórico y de pruebas pilotos en el contexto peruano. En ambos casos, sea partiendo de supuestos o de diagnósticos inconsistentes, al final no son más que diatribas de lucro que históricamente han engordado a los malos administradores del estado y de cuyas comodidades desprenderse no será fácil. Aun así, no hay que juzgar a las personas, sino a los enfoques que gobiernan el cerebro de aquellas "mentes brillantes". 
En casi doscientos años de vida republicana, el pueblo peruano ha sufrido los estragos de una educación utilitarista, desacertada. Dos factores saltan a la vista: el desconocimiento real del hecho educativo y la ausencia de fundamentos pertinentes genuinos, científicamente construidos, validados y probados en el mismo hecho por agentes educativos. Por ello, sin temor a equivocación, conviene la conjugación dialéctica de base y de fundamento. El eventual desconocimiento de dicha relación sea por ignorancia u omisión voluntaria tirada por la fuerza de enfoques metodológicos reduccionistas, conlleva a rotundos fracasos.

En todo caso, metodológicamente hablando, debemos asumir la comprensión de los objetos y fenómenos de la realidad como resultados y procesos unitarios e interactivos: base y fundamento, son temas de primer orden. El olvido de uno de estos aspectos o la sobrevaloración en desmedro de uno de los dos, podrían conducirnos al callejón sin salida de la muerte científica. Cualquiera que fuera la actividad humana, es más viable entenderla desde el equilibro entre base y fundamento. Sin el equilibrio, la condena al reduccionismo es inevitable.

\section{Teoría y práctica en la producción de conocimientos científicos}

El tránsito de la transmisión mecánica del conocimiento hacia la producción crítica y creativa, implica conjugar crítica y creativamente la teoría y la práctica.

La práctica es un manantial de conocimientos, en tanto sitúa al hombre ante sistemáticos conflictos en cuya solución descubre nuevos conocimientos, procedimientos y posibilita el desarrollo de capacidades. No obstante, este importante papel de la práctica, como manantial inagotable de conocimientos, sin teoría no constituye móvil de conocimientos. La teoría dirige a la práctica, posee su propia independencia, gracias a lo cual es capaz de adelantarse a la realización de algún acontecimiento. La ciencia es el resultado de la interacción de la teoría y la práctica, avanza, por ambas vías, y se confirma en cada realización teórica y práctica.

Lograr el balance adecuado entre la teoría y la práctica es responsabilidad que preocupa a la escuela como institución social que responde por potenciar el desarrollo personal, de cada ser social, al máximo de sus posibilidades individuales. Este balance puede peligrar si no se atienden dichos entes en su relación complementaria y de exclusión. Absolutizar el aspecto teórico conduce a un conocimiento muerto, sin objetivo, desprovisto de utilidad. Un aprendizaje así logrado es estéril y en última instancia no es tal aprendizaje ya que carece de objetivo y por tanto es arbitrario. Si por el contrario se hiperboliza lo práctico, se produce un aprendizaje utilitarista, pragmático, fenoménico y por tanto incapaz de ser utilizado ante nuevas condiciones. En la institución docente esta relación se particulariza en el vínculo de la escuela con la vida, lo que trasciende al nexo entre el estudio y el trabajo. 
Desde el diseño curricular hasta la tarea docente debe atenderse al importante vínculo entre lo teórico y lo práctico.

\section{Perspectivas}

El educador peruano busca romper con el empirismo. Quizás por ahora no está en capacidad de lograrlo sea porque no ha sido formado en una concepción ni en un espíritu científico, ni ha recibido las herramientas intelectuales de tipo teórico-metodológico que puedan permitírselo. Pero tiene voluntad de cambio y de progreso. Aspira a elevar su nivel profesional, está sincera y profundamente preocupado por la formación y el aprendizaje de sus estudiantes, pero a la vez vive atrapado por sus propias limitaciones y no encuentra la orientación justa y adecuada para superar esa situación. Entonces, en muchos casos, es inducido al escepticismo, al conformismo y a la apatía cognoscitiva, con grave perjuicio no sólo para él como ser humano, sino también para los estudiantes colocados bajo su tutela. La evidencia concreta demuestra que los docentes están situados, contra su voluntad y sus deseos reales, en una situación profesional profundamente desventajosa no sólo en términos de consideración social, sino también en cuanto al propio desempeño de su actividad docente, por presentar vacíos en su formación pedagógica y carecer de oportunidades para su actualización científica. Pero demuestra también, por un lado, que hay educadores preocupados por diseñar una línea cognoscitiva en concordancia con el desarrollo de la ciencia contemporánea, por buscar conocimientos e informaciones susceptibles de elevar la calidad de su desempeño en el aula, por desplegar esfuerzos individuales y colectivos en procura de encontrar vías precisas para favorecer el desarrollo de sus educandos y, en fin, por agruparse para pensar y luchar por una educación científica al servicio de las mayorías nacionales, y que, por el otro, revela la existencia de docentes agobiados por la frustración, aplastados por las circunstancias concretas de la dura vida cotidiana, enceguecidos por la telaraña de la ideología dominante que exige el sometimiento pasivo al caos social vigente, y que han renunciado a pensar, a ejercer sus derechos a combatir por sus convicciones e ideales. 


\section{REFERENCIAS}

Cuenca, R. (2015) Educación Universitaria en el Perú. IEP.

Castro, L. (2007). Elementos de psicopedagogía docente (2da.Ed) Lima: EDUCAP

Castro, L. (2005). Diccionario de ciencias de la educación (2da.Ed) Lima: EDUCAP

Ferrater, J (1991) Diccionario de Filosofía. Madrid: Aula Santillana, S.A.

Fiallo, J (2008) La investigación pedagógica una vía para elevar la calidad educativa. Lima: Ruta pedagógica.

Lefebvre. H (1958) El marxismo (6ta.Ed) Buenos Aires: EUA.

Selsam, H. (2006) Qué es la filosofía. California: Publisher.

Minedu (2012) Marco del Buen Desempeño Docente. Lima. 